\title{
PERMUTATION-PARTITION PAIRS: A COMBINATORIAL GENERALIZATION OF GRAPH EMIBEDDINGS
}

\begin{abstract}
BY
SAUL STAHL ${ }^{1}$

ABSTRACT. Permutation-partition pairs are a purely combinatorial generalization of graph embeddings. Some parameters are defined here for these pairs and several theorems are proved. These results are strong enough to prove virtually all the known theoretical informaton about the genus parameter as well as a new theorem regarding the genus of the amalgamation of two graphs over three points.
\end{abstract}

Introduction. Like many other branches of graph theory, the subject of graph embeddings has its roots in the 4-color problem. This problem was given official recognition by Cayley in 1878 [C] at a time when Riemann surfaces were still relatively new and the subject of much active research. It was only natural that someone should pose the analogous question for these new surfaces, as indeed Heawood did in 1890 [H1]. For an account of the exciting history of this colorful problem, culminating in its eventual solution by Ringel and Youngs in 1968 the reader is referred to [R].

It was very quickly observed by the originators of this new coloring problem that it has a natural dual problem which can be formulated as that of determining $\gamma\left(K_{n}\right)$-the minimum of all the genera of the Riemann surfaces on which the complete graph $K_{n}$ can be embedded. This, of course, generalizes to the problem of evaluating $\gamma(G)$-the genus of the graph $G$. In its turn, this generalization can be given a purely combinatorial formulation which involves the estimation of the number of cycles in the disjoint cycle decomposition of the product $E \circ R$, where $E$ is an involution determined by the edges of $G$, and $R$ is a permutation determined by the vertices of $G[\mathrm{~W}]$. It was noted by the author that if the constraints of this problem are relaxed, in that $E$ is not necessarily restricted to be an involution, then this extended problem has some very natural applications to the estimation of the genus of the amalgamation of two graphs. This extended combinatorial problem and its applications to the genus of graphs are the subject matter of this paper.

Accordingly, there are two parts to this paper. The first deals with the above combinatorial problem without any reference to its topological origins. The second part applies the results of the first to the derivation of a variety of known and new theorems in the theory of graph embeddings.

Received by the editors February 8, 1979 and, in revised form, June 25, 1979; presented to the Society, January 25, 1979.

AMS (MOS) subject classifications (1970). Primary 05C10.

${ }^{1}$ This investigation was supported by University of Kansas General Research Allocation \#3496-x0-0038. 
1. Permutation-partition pairs. In this report the product of permutations should be read from left to right. The disjoint cycle decomposition of a permutation $P$ is denoted by $P: \sigma_{1} \sigma_{2} \ldots \sigma_{r}$. The number of orbits in the disjoint cycle decomposition of a permutation $P$ is denoted by $\|P\|$. We denote by $a P$ the image of $a$ under the action of the permutation $P$. A $P P(n, k)$ pair $(P, \Pi)$ consists of a permutation $P$ and a partition $\Pi=\left\{\Pi_{i}\right\}_{i=1}^{k}$ defined on some common finite set $S$ with $n$ elements. These elements will be referred to as bits. It is to be understood that none of the $\Pi_{i}$ is empty. If two bits $a$ and $b$ belong to the same member of $\Pi$ we write $a \equiv b$ $(\bmod \Pi)$. By $S(\Pi)$ we denote the set of permutations on $S$, each of whose orbits is in fact a member of $\Pi$. The maximum orbiticity $\mu(P, \Pi)$ of the pair $(P, \Pi)$ is defined by $\mu(P, \Pi)=\max \{\|P Q\| \mid Q \in S(\Pi)\}$. It is this maximum orbiticity that is the subject matter of the first half of this report.

Let $(P, \Pi)$ be a $P P(n, k)$ pair, and suppose $\left\{a_{i}\right\}_{i=1}^{m}$ is a set of bits. We say that the $P P\left(n-m, k^{\prime}\right)$ pair $\left(P^{\prime}, \Pi^{\prime}\right)$ is obtained from $(P, \Pi)$ by suppressing $\left\{a_{1}, \ldots, a_{m}\right\}$ if the cyclic factors of $P^{\prime}$ are obtained from those of $P$ by deleting all the bits $\left\{a_{i}\right\}_{i=1}^{m}$, and the members of $\Pi^{\prime}$ are obtained from those of $\Pi$ in the same manner. Should any member of $\Pi$ consist only of bits to be deleted it makes no contribution to $\Pi^{\prime}$,

ExAmple 1. $P=(1)(23)(45678)(9 a), \Pi=\{\{1,2\},\{3,4,5,6\},\{7,8,9, a\}\}$. By suppressing $\{1,2,6\}$ we obtain the pair $\left(P^{\prime}, \Pi^{\prime}\right)$ where $P^{\prime}=(3)(4578)(9 a), \Pi^{\prime}=$ $\{\{3,4,5\},\{7,8,9, a\}\}$.

Let $(P, \Pi)$ be a $P P(n, k)$ pair. Then $\kappa(P, \Pi)$ denotes the number of orbits of $P$ that are contained in some member of $\Pi$. The switching number $\sigma(P, \Pi)$ is the number of bits $a$ such that $a \neq a P(\bmod \Pi)$. Finally, if $k=3$ we define the winding number $\omega(P, \Pi)$ as follows. For each bit $a$, let $i(a)$ and $j(a)$ be defined by

$$
a \in \Pi_{i(a)}, \quad a P \in \Pi_{j(a)} .
$$

Now set

$$
\omega(P, \Pi)=\frac{1}{3}\left|\sum_{a \in S}[j(a)-i(a)]\right| .
$$

EXAMPle 2. If $P=(1)(23)(6)(4 a 7 b 8)(59 c d)$ and $\Pi=\{\{1,2,3,4,5\}$, $\{6,7,8,9\},\{a, b, c, d\}\}$, then $\kappa(P, \Pi)=3, \sigma(P, \Pi)=8, \omega(P, \Pi)=0$.

Two permutations $P$ and $Q$ of the set $T$ are said to be disjoint if for all $a \in T$, $a P \neq a$ implies that $a Q=a$ and vice versa, $a Q \neq a$ implies that $a P=a$. Observe that if $Q \in S(\Pi)$ then $P$ and $P Q$ map the bit $a$ into the same member of $\Pi$. This makes the proof of the following facts immediate.

Lemma 3. Let $(P, \Pi)$ and $\left(P_{i}, \Pi\right), i=1,2$, be $P P(n, k)$ pairs such that $P=P_{1} P_{2}$, $P_{1}$ and $P_{2}$ are disjoint, and suppose $Q \in S(\Pi)$. Then

$$
\begin{aligned}
& \sigma(P Q, \Pi)=\sigma(P, \Pi)=\sigma\left(P_{1}, \Pi\right)+\sigma\left(P_{2}, \Pi\right), \\
& \omega(P Q, \Pi)=\omega(P, \Pi)=\omega\left(P_{1}, \Pi\right)+\omega\left(P_{2}, \Pi\right) \quad \text { if } k=3 .
\end{aligned}
$$

A pair $(P, \Pi)$ is said to be decomposable into the pairs $\left\{\left(P_{i}, \Pi^{(i)}\right)\right\}_{i=1}^{m}$ if $\left\{\Pi^{(i)}\right\}_{i=1}^{m}$ $(m \geqslant 2)$ is a partition of $\Pi, P_{i} \in S\left(\Pi^{(i)}\right), i=1,2, \ldots, m$, and $P=P_{1} P_{2} \ldots P_{m}$. If $(P, \Pi)$ has no decompositions, then it is indecomposable. 
Example 4. If $\Pi=\{\{1,2,3\},\{4,5,6\},\{a, b, c\}\}, P=(1453)(2)(6)(a b)(c), Q=$ $(1)(a)(6)(24)(5 b)(3 c)$, then $(Q, \Pi)$ is indecomposable and $(P, \Pi)$ decomposes into $\left(P_{1}, \Pi^{(1)}\right)$ and $\left(P_{2}, \Pi^{(2)}\right)$ where

$$
\begin{array}{ll}
P_{1}=(1453)(2)(6), & \Pi^{(1)}=\{\{1,2,3\},\{4,5,6\}\}, \\
P_{2}=(a b)(c), & \Pi^{(2)}=\{\{a, b, c\}\} .
\end{array}
$$

Lemmas 5 and 6 provide reduction methods to be used in subsequent theorems.

Lemma 5. Let $(P, \Pi)$ be a $P P(n, k)$ pair and suppose that for some bits $a, b$, $b=a P \neq a$ and $a \equiv b(\bmod \Pi)$. If $\left(P^{\prime}, \Pi^{\prime}\right)$ is the pair obtained by suppressing $a$ in $(P, \Pi)$, then it is a $P P(n-1, k)$ pair and

$$
\begin{array}{ll}
\mu(P, \Pi)=\mu\left(P^{\prime}, \Pi^{\prime}\right)+1, & \kappa(P, \Pi)=\kappa\left(P^{\prime}, \Pi^{\prime}\right), \\
\sigma(P, \Pi)=\sigma\left(P^{\prime}, \Pi^{\prime}\right), & \omega(P, \Pi)=\omega\left(P^{\prime}, \Pi^{\prime}\right) \quad \text { if } k=3 .
\end{array}
$$

Moreover, if $(P, \Pi)$ is indecomposable, so is $\left(P^{\prime}, \Pi^{\prime}\right)$.

Proof. Since $a \neq b$ and $a$ and $b$ are in the same member of $\Pi$ it follows that $\left(P^{\prime}, \Pi^{\prime}\right)$ is a $P P(n-1, k)$ pair. That $\kappa(P, \Pi)=\kappa\left(P^{\prime}, \Pi^{\prime}\right)$ follows from the fact that $a$ and $b$ are in the same orbit of $P$ and the same member of $\Pi$. For the same reason the indecomposability of $(P, \Pi)$ is inherited by $\left(P^{\prime}, \Pi^{\prime}\right)$. Since $a \equiv a P(\bmod \Pi)$, the bit $a$ only makes a zero contribution to both $\sigma$ and $\omega$. Consequently, $\sigma(P, \Pi)=$ $\sigma\left(P^{\prime}, \Pi^{\prime}\right)$ and $\omega(P, \Pi)=\omega\left(P^{\prime}, \Pi^{\prime}\right)$. It only remains to prove the equality involving $\mu$.

Let $Q \in S(\Pi)$ and $Q^{\prime} \in S\left(\Pi^{\prime}\right)$ be such that $\mu(P, \Pi)=\|P Q\|$ and $\mu\left(P^{\prime}, \Pi^{\prime}\right)=$ $\left\|P^{\prime} Q^{\prime}\right\|$. Let $\tilde{Q} \in S(\Pi)$ be defined by stipulating that it agree with $Q^{\prime}$ everywhere except at $b$, and $b \tilde{Q}=a, a \tilde{Q}=b Q^{\prime}$. In other words, $\tilde{Q}$ is obtained from $Q^{\prime}$ by inserting $a$ to the right of $b$. Then, $P \tilde{Q}=P^{\prime} Q^{\prime}(a)$ and so $\mu(P, \Pi)>\mu\left(P^{\prime}, \Pi^{\prime}\right)+1$.

To prove the opposite inequality, suppose first that $b Q=c \neq a$. Since $a$ is in the same cyclic factor of $Q$ as $b$ and $c$, it follows that $\bar{Q}=Q(a b)(a c) \in S(\Pi)$. Moreover, $a P Q(a b)=c$, and it is well known [S] that in general $\|T(x y)\|=\|T\| \pm$ 1 according to whether $x$ and $y$ do or do not belong to the same orbit of $T$. Hence,

$$
\|P \bar{Q}\|=\|P Q(a b)(a c)\|=\|P Q(a b)\|+1>\|P Q\| \text {. }
$$

From the maximality of $\|P Q\|$ it now follows that $\mu(P, \Pi)=\|P \bar{Q}\|$ where $\bar{Q}$ has the property that $b \bar{Q}=a$. If $\bar{Q}^{\prime}$ is obtained from $\bar{Q}$ by suppressing $a$, then $P \bar{Q}=P^{\prime} \bar{Q}^{\prime}(a)$. Hence $\mu(P, \Pi)=\|P \bar{Q}\|=\left\|P^{\prime} \bar{Q}^{\prime}\right\|+1<\mu\left(P^{\prime}, \Pi^{\prime}\right)+1$.

Finally, if it so happens that $b Q=a$, then we need merely replace $\bar{Q}$ by $Q$ in the above argument.

The proof of the following lemma is a straightforward adaptation of the previous proof and hence its details are omitted.

LemMa 6. Let $(P, \Pi)$ be a $P P(n, k)$ pair and suppose that $P$ has the singleton $(a)$ as an orbit. If $\left(P^{\prime}, \Pi^{\prime}\right)$ is the pair obtained from $(P, \Pi)$ by suppressing $a$, then

$$
\begin{aligned}
& \kappa(P, \Pi)=\kappa\left(P^{\prime}, \Pi^{\prime}\right)+1, \quad \sigma(P, \Pi)=\sigma\left(P^{\prime}, \Pi^{\prime}\right), \\
& \omega(P, \Pi)=\omega\left(P^{\prime}, \Pi^{\prime}\right) \quad \text { if } k=3 .
\end{aligned}
$$


Moreover, if $(P, \Pi)$ is indecomposable, then $\left(P^{\prime}, \Pi^{\prime}\right)$ is an indecomposable $P P(n-1$, k) pair, satisfying $\mu(P, \Pi)=\mu\left(P^{\prime}, \Pi^{\prime}\right)$.

TheOREM 7. If $(P, \Pi)$ is a $P P(n, 1)$ pair then $\mu(P, \Pi)=n-\|P\|+1$.

Proof. By induction on $n$. If $n=1$, or if $n>1$ and $\|P\|=n$, the theorem is immediate. Assume the theorem to hold for all $P P(m, 1)$ pairs $m<n$. If $n>1$ and $\|P\|<n$, then some orbit of $P$ has cardinality at least two and hence there exist elements $a$ and $b$ satisfying the hypotheses of Lemma 5. Using the notation of this lemma, it follows from the induction hypothesis that

$$
\mu(P, \Pi)=\mu\left(P^{\prime}, \Pi^{\prime}\right)+1=n-1-\|P\|+1+1=n-\|P\|+1
$$

and the induction process is complete.

Corollary 8. If $(P, \Pi)$ is decomposable into $k P P\left(s_{j}, 1\right)$ pairs such that $\sum_{j=1}^{k} s_{j}=$ $n$, then $\mu(P, \Pi)=n-\|P\|+k$.

We now state without proof three facts about permutations. The first of these is due to A. M. Gleason and a proof can be found in [H2]. The others follow easily from the first.

Proposition 9. (a) Every even permutation of the symmetric group $S_{n}$ is the product of at most two cycles of length $n$.

(b) If $P$ is an arbitrary permutation of $S_{n}$ and $p$ is an arbitrary integer greater than 1 , then there exist cyclic permutations $C_{1}, \ldots, C_{p}$, each of order $n$, such that $P C_{1} \ldots C_{P}$ is either the identity or else a transposition.

(c) If $P$ is an arbitrary permutation of $S_{n}$, then there exists a cycle $C$ of order $n$ such that $\|P \circ C\| \leqslant 2$.

TheOrem 10. Let $(P, \Pi)$ be an indecomposable $P P(n, 2)$ pair. Then

$$
n-\kappa(P, \Pi)-\frac{1}{2} \sigma(P, \Pi)-1 \leqslant \mu(P, \Pi)<n-\kappa(P, \Pi)-\frac{1}{2} \sigma(P, \Pi) .
$$

Proof. By induction on $n$. If $n=2$ then $\mu(P, \Pi)=1$ and the theorem holds. Assume the theorem has been verified for all $P P(m, 2)$ pairs, $m<n$. If $(P, \Pi)$ has a bit $a$ satisfying the hypotheses of Lemmas 5 or 6 , then (2) follows by applying the induction hypothesis to the derived pair $\left(P^{\prime}, \Pi^{\prime}\right)$. We may therefore assume now that $(P, \Pi)$ has no such bits $a$ and hence the cyclic factors of $P$ alternate between bits of $\Pi_{1}$ and $\Pi_{2}$. Necessarily, $\kappa(P, \Pi)=0, \sigma(P, \Pi)=n$, and $\left|\Pi_{1}\right|=\left|\Pi_{2}\right|=n / 2$.

Let $Q: \tau_{1} \tau_{2}$ be an arbitrary permutation of $S(\Pi)$, where $\tau_{i}$ is a cyclic permutation of $\Pi_{i}, i=1,2$. Just like $P$, the product $P Q$ maps elements of $\Pi_{1}$ into $\Pi_{2}$ and vice versa. Hence $(P Q)^{2}=K_{1} K_{2}$ where $K_{i}$ is a permutation on $\Pi_{i}, i=1,2$. Moreover, each orbit of $P Q$ is the union of an orbit of $K_{1}$ and an orbit of $K_{2}$; hence $\|P Q\|=\left\|K_{1}\right\|$. Note that

$$
K_{1}=\left.(P Q P Q)\right|_{\Pi_{1}}=\left.\left(P^{2} \circ P^{-1} Q P \circ Q\right)\right|_{\Pi_{1}}=\left.\left.\left(P^{2}\right)\right|_{\Pi_{1}} \circ\left(P^{-1} \tau_{2} P\right)\right|_{\Pi_{1}} \circ \tau_{1} .
$$

As $\tau_{2}$ varies over all the cyclic permutations of $\Pi_{2}$, the product $\left.\left(P^{-1} \tau_{2} P\right)\right|_{\Pi_{1}}$ varies over all the cyclic permutations of $\Pi_{1}$, and vice versa. Now, by Proposition 9 , there exist cycles $\tau_{1}$ and $\tau_{2}$ such that $K_{1}$ is either the identity on $\Pi_{1}$ or else it is a 
transposition. In either case, $\left\|K_{1}\right\|>n / 2-1$. If $Q$ is the element of $S(\Pi)$ determined by this choice of $\tau_{2}$ and $\tau_{1}$, then

$$
\mu(P, \Pi) \geqslant\|P Q\|=\left\|K_{1}\right\|>n / 2-1 .
$$

On the other hand, since every orbit of $P Q$ alternates elements of $\Pi_{1}$ and $\Pi_{2}$, it follows that each such orbit contains at least two elements and so $n / 2>\mu(P, \Pi)$. Recalling that in this case we have $\kappa=0$ and $\sigma=n$, the induction process is complete.

COROllary 11. If $(P, \Pi)$ is a $P P(n, 3)$ pair which has a decomposition $\left(P_{i}, \Pi^{(i)}\right)_{i=1}^{m}$, where each $\left(P_{i}, \Pi^{(i)}\right)$ is itself indecomposable, then

$$
\begin{gathered}
\mu(P, \Pi)=n-\kappa(P, \Pi)+3 \text { if } m=3, \\
n-\kappa(P, \Pi)-\frac{1}{2} \sigma(P, \Pi) \leqslant \mu(P, \Pi) \leqslant n-\kappa(P, \Pi)-\frac{1}{2} \sigma(P, \Pi)+1 \text { if } m=2 .
\end{gathered}
$$

In order to eliminate the need for superscripts in the sequel, the following notational conventions are established here. If $(P, \Pi)$ is a $\operatorname{PP}(n, 3)$ pair, and $\Pi=\left\{\Pi_{i}\right\}_{i=1}^{3}$, then

$\Pi_{1}=A=\left\{a_{1}, a_{2}, \ldots\right\}, \quad \Pi_{2}=B=\left\{b_{1}, b_{2}, \ldots\right\}, \quad \Pi_{3}=C=\left\{c_{1}, c_{2}, \ldots\right\}$.

We now describe a reduction procedure which is essential to the evaluation of the maximum orbiticity of $P P(n, 3)$ pairs. If $(P, \Pi)$ is a $P P(n, 3)$ pair with (possibly reindexed) bits such that $a_{1} P=b_{1}$ and $b_{2} P=a_{2}$, then $\bar{P}=P\left(a_{1} a_{2}\right)\left(b_{1} b_{2}\right)$ has $\left(a_{1} b_{2}\right)$ as an orbit. The $P P(n-2, k)$ pair, $(k<3)$ obtained by suppressing $\left\{a_{1}, b_{2}\right\}$ in $(\bar{P}, \Pi)$ is referred to as the 2-reduced pair. For instance, if we set in Example 2, $a_{1}=a, a_{2}=c, b_{1}=7, b_{2}=9$, then the 2-reduction yields the pair $\left(P^{\prime}, \Pi^{\prime}\right)$ where

$$
P^{\prime}=(1)(23)(6)(b 84 c d 57)
$$

and

$$
\Pi^{\prime}=\{\{1,2,3,4,5\},\{6,7,8\},\{b, c, d\}\}
$$

Lemma 12. Suppose the $P P(n, k)$ pair $(P, \Pi)$ is 2-reduced to $\left(P^{\prime}, \Pi^{\prime}\right)$. Then

$$
\begin{gathered}
\kappa\left(P^{\prime}, \Pi^{\prime}\right) \geqslant \kappa(P, \Pi) \geqslant \kappa\left(P^{\prime}, \Pi^{\prime}\right)-2, \\
\mu(P, \Pi)>\mu\left(P^{\prime}, \Pi^{\prime}\right)+1, \\
\sigma(P, \Pi)=\sigma\left(P^{\prime}, \Pi^{\prime}\right)+2, \\
\omega(P, \Pi)=\omega\left(P^{\prime}, \Pi^{\prime}\right) \quad \text { if } k=3 .
\end{gathered}
$$

Proof. Let $X, Y, Z, W$ denote possibly empty strings of bits. Since a 2-reduction does apply to $(P, \Pi)$, one of the following must hold. Either

$$
P:\left(X a_{1} b_{1} Y b_{2} a_{2}\right) \ldots \text { and } P^{\prime}:\left(X a_{2}\right)\left(Y b_{1}\right) \ldots
$$

or

$$
P:\left(X a_{1} b_{1}\right)\left(Y b_{2} a_{2}\right) \ldots \text { and } P^{\prime}:\left(X a_{2} Y b_{1}\right) \ldots
$$

Let $Q^{\prime}:\left(Z a_{2}\right)\left(W b_{1}\right) \ldots \in S\left(\Pi^{\prime}\right)$ be such that $\left\|P^{\prime} Q^{\prime}\right\|=\mu\left(P^{\prime}, \Pi^{\prime}\right)$, and define $Q$ : $\left(Z a_{2} a_{1}\right)\left(W b_{1} b_{2}\right) \ldots \in S(\Pi)$. It is then easily verified that $P Q$ has all the orbits of $P^{\prime} Q^{\prime}$ plus the additional orbit of $\left(a_{1} b_{2}\right)$. Hence $\mu(P, \Pi)>\mu\left(P^{\prime}, \Pi^{\prime}\right)+1$. 
That $\omega(P, \Pi)=\omega\left(P^{\prime}, \Pi^{\prime}\right)$ follows, for $k=3$, from the observations that the expansion of (1) for $\left(P^{\prime}, \Pi^{\prime}\right)$ differs from that of $(P, \Pi)$ merely by the quantity $j\left(a_{1}\right)-i\left(a_{1}\right)+j\left(b_{2}\right)-i\left(b_{2}\right)=i\left(b_{1}\right)-i\left(a_{1}\right)+i\left(a_{2}\right)-i\left(b_{2}\right)=0$. A similar analysis shows that, in general, $\sigma(P, \Pi)=\sigma\left(P^{\prime}, \Pi^{\prime}\right)+2$.

The inequalities involving $\kappa$ are clear. Note, for instance, that $\kappa(P, \Pi)<$ $\kappa\left(P^{\prime}, \Pi^{\prime}\right)$ only if the 2-reduction is of type (3) and then only if $X \subseteq A$ or $Y \subseteq B$ or both.

TheOREM 13. If $(P, \Pi)$ is an indecomposable $P P(n, 3)$ pair, then

$$
\begin{aligned}
n-\kappa(P, \Pi)-\frac{1}{2} \sigma(P, \Pi)- & \frac{1}{2} \omega(P, \Pi)-3<\mu(P, \Pi) \\
& \leqslant n-\kappa(P, \Pi)-\frac{1}{2} \sigma(P, \Pi)-\frac{1}{2} \omega(P, \Pi) .
\end{aligned}
$$

Proof. The theorem is proved by induction on $n$. If $n=3,4$ the theorem may be verified easily by testing all the indecomposable pairs. Assume now that the theorem has been verified for all indecomposable $P P(m, 3)$ pairs where $3<m<n$ and $n \geqslant 5$.

Suppose the indecomposable $(P, \Pi)$ possesses a bit $a$ satisfying the hypotheses of Lemma 5 or 6 . Then (5) follows from the application of the induction hypothesis to the derived indecomposable pair $\left(P^{\prime}, \Pi^{\prime}\right)$. Hence we may, and do, henceforth assume that for any bit $a, a \neq a P(\bmod \Pi)$. Necessarily, $\kappa(P, \Pi)=0$ and $\sigma(P, \Pi)$ $=n$. We now prove the upper bound of (5). Suppose $Q \in S(\Pi)$ is such that $\mu=\mu(P, \Pi)=\|P Q\|$. Suppose further that $P Q$ has $\mu_{i}$ orbits of cardinality $i$, $i=2,3, \ldots$, (clearly $P Q$ has no singletons as orbits). Now, if $C$ is a cyclic permutation of order $m$, then clearly $\omega(C, \Pi)<m-2$. Hence it follows from several applications of Lemma 3 that

$$
\begin{aligned}
\omega & =\omega(P, \Pi)=\omega(P Q, \Pi)<\sum_{i=2}^{\infty}(i-2) \mu_{i} \\
& =\sum_{i=2}^{\infty} i \mu_{i}-2 \sum_{i=2}^{\infty} \mu_{i}=n-2 \mu .
\end{aligned}
$$

Hence

$$
\mu \leqslant \frac{n-\omega}{2}=n-\frac{\sigma}{2}-\frac{\omega}{2}=n-\kappa-\frac{\sigma}{2}-\frac{\omega}{2} .
$$

This establishes the upper bound of (5). The lower bound is proved by an application of Lemma 12. Suppose $(P, \Pi)$ is 2-reduced to the $\operatorname{PP}(n-2, k)$ pair $\left(P^{\prime}, \Pi^{\prime}\right)$. Since Lemmas 5 and 6 do not apply to $(P, \Pi)$ it follows that $\kappa\left(P^{\prime}, \Pi^{\prime}\right)=$ $\kappa(P, \Pi)$ (see the last paragraph of proof of Lemma 12). If $\left(P^{\prime}, \Pi^{\prime}\right)$ is also indecomposable, then by the induction hypothesis,

$$
\begin{aligned}
\mu(P, \Pi) \geqslant & \mu\left(P^{\prime}, \Pi^{\prime}\right)+1>n-2-\kappa\left(P^{\prime}, \Pi^{\prime}\right) \\
& -\frac{1}{2} \sigma\left(P^{\prime}, \Pi^{\prime}\right)-\frac{1}{2} \omega\left(P^{\prime}, \Pi^{\prime}\right)-3+1 \\
= & n-\kappa(P, \Pi)-\frac{1}{2} \sigma(P, \Pi)-\frac{1}{2} \omega(P, \Pi)-3 .
\end{aligned}
$$

If $\left(P^{\prime}, \Pi^{\prime}\right)$ is decomposable, then the lower bound for $\mu\left(P^{\prime}, \Pi^{\prime}\right)$ must come from Corollary 11. Note that if $m=2$, then $\omega\left(P^{\prime}, \Pi^{\prime}\right)=0$ whereas if $m=3$, then 
$\sigma\left(P^{\prime}, \Pi^{\prime}\right)=\omega\left(P^{\prime}, \Pi^{\prime}\right)=0$ and in either case the above inequality holds.

Hence it may now be assumed that Lemma 12 cannot be applied to $(P, \Pi)$. Consequently, bearing in mind that $a \neq a P(\bmod \Pi)$ for every bit $a, P$ can be explicitly displayed. In fact, we shall show that modulo a permutaton of the symbols $a, b, c, P$ must belong to one of the following sets of permutations.

(I) $\left(\Pi_{i}\right) P=\Pi_{i+1}, i=1,2,3$ (addition modulo 3 ).

$$
\begin{aligned}
P_{1} & =\left(a_{1} b_{1}\right), \\
P_{2} & =\left(a_{1} b_{1}\right)\left(b_{2} c_{1}\right), \\
P_{3} & =\left(a_{1} b_{1}\right)\left(b_{2} c_{1}\right)\left(c_{2} a_{2}\right), \\
P_{4} & =\left(a_{1} b_{1} a_{2} b_{2}\right), \\
P_{5} & =\left(a_{1} b_{1} a_{2} b_{2}\right)\left(b_{3} c_{1}\right), \\
P_{6} & =\left(a_{1} b_{1} a_{2} b_{2}\right)\left(b_{3} c_{1}\right)\left(c_{2} a_{3}\right), \\
P_{7} & =\left(a_{1} b_{1} a_{2} b_{2}\right)\left(b_{3} c_{1} b_{4} c_{2}\right), \\
P_{8} & =\left(a_{1} b_{1} a_{2} b_{2}\right)\left(b_{3} c_{1} b_{4} c_{2}\right)\left(c_{3} a_{3}\right), \\
P_{9} & =\left(a_{1} b_{1} a_{2} b_{2}\right)\left(b_{3} c_{1} b_{4} c_{2}\right)\left(c_{3} a_{3} c_{4} a_{4}\right), \\
P_{10} & =\left(a_{1} b_{1} c_{1} a_{2} b_{2}\right), \\
P_{11} & =\left(a_{1} b_{1} c_{1} a_{2} c_{2} a_{3} b_{2}\right), \\
P_{12} & =\left(a_{1} b_{1} c_{1} b_{2}\right)\left(a_{2} c_{2}\right), \\
P_{13} & =\left(a_{1} b_{1} c_{1} b_{2}\right)\left(a_{2} c_{2} a_{3} c_{3}\right), \\
P_{14} & =\left(a_{1} b_{1} c_{1} b_{2}\right), \\
P_{15} & =\left(a_{1} b_{1} c_{1} b_{2} c_{2}\right), \\
P_{16} & =\left(a_{1} b_{1} c_{1} b_{2} c_{2} a_{2} c_{3} a_{3} b_{3}\right)
\end{aligned}
$$

For suppose first that no cyclic factor of $P$ intersects all three members of $\Pi$. Then, each orbit alternates between two members of $\Pi$. Since Lemma 12 does not apply to $P$, at most one orbit intersects both of a given pair of members of $\Pi$. Finally, since Lemma 12 does not apply, each orbit of $P$ contains at most four bits. Hence $P=P_{i}, 1<i<9$.

If some cyclic factor $\varphi$ of $P$ does intersect all three members of $\Pi$, then we assume without loss of generality that $\varphi=\left(a_{1} b_{1} c_{1} x y z w v u \ldots\right)$. If $x$ were in $C$, then the reduction of Lemma 5 would apply to $P$ with $a=c_{1}$ and $b=x$. Since we know this not to be the case it follows that $x \in A \cup B$.

If $x \in A$, then since the $P$ is not 2-reducible, it follows that every other cyclic factor of $P$ has the periodic structure of the permutations belonging to set (I). Should $\varphi$ itself have the same form we are done. Otherwise, we may assume that after a suitable relabelling, $\varphi=\left(a_{1} b_{1} c_{1} a_{2} b_{2} z \ldots\right)$ with $z \notin C$ or else $\varphi=$ $\left(a_{1} b_{1} c_{1} a_{2} c_{2} z w v \ldots\right)$, and in either case $P$ can have no other cyclic factors but $\varphi$. In the first of these two cases, if $z=a_{1}$, then $P=P_{10}$; if $z \in A-\left\{a_{1}\right\}$, then Lemma 12 would apply to the pairs $\left(a_{1} b_{1} \ldots b_{2} z \ldots\right)$, whereas if $z \in B$, then Lemma 5 would be applicable, neither of which is possible. Proceeding with the second case, 
if $z=a_{1}$, again $P=P_{10}$. Clearly $z \notin C$ since otherwise Lemma 5 would apply. The nonapplicability of Lemma 12 implies that $z \notin B$ and hence $z=a_{3}$. The above assumption that Lemmas 5 and 6 do not apply to $P$ forces $w$ to belong to $B$. However, any choice for $v$ except $v=a_{1}$ cannot be followed by a consistent choice for $u$. Hence $v=a_{1}$ and $P=P_{11}$.

Finally, if $x \in B$, then necessarily $\varphi=\left(a_{1} b_{1} c_{1} b_{2} y z w v \ldots\right)$. If $\theta$ is any other cyclic factor of $P$ then it cannot contain a $b_{p}$ since, whatever the value of $b_{p} \theta$, it might be used to reduce $P$ by an application of Lemma 6 or Lemma 12. Lemmas 5 and 12 in fact force us to conclude that any such $\theta$ must in fact be either a transposition $\left(a_{p} c_{q}\right)$ or a four-cycle $\left(a_{p} c_{q} a_{r} c_{s}\right)$ and that there can be at most one of these in $P$. If $P$ does contain such a $\theta$, then $\varphi$ cannot contain a consecutive pair of the form $(\ldots a c \ldots)$ or $(\ldots c a \ldots)$. This is sufficient information to conclude that $\varphi=\left(a_{1} b_{1} c_{1} b_{2}\right)$ and hence $P=P_{12}$ or $P_{13}$. On the other hand, if $P$ contains no such $\theta$ then $P=P_{11}, P_{14}, P_{15}$ or $P_{16}$.

We conclude the proof by showing that the indecomposable pairs coming from (I) and (II) also satisfy the lower bound of (5). For set (II), the verification is done by direct calculations and the details are left to the reader. If $(P, \Pi)$ is of type (I), let $Q: \tau_{1} \tau_{2} \tau_{3}$ be an arbitrary permutation of $S(\Pi)$ where each $\tau_{i}$ is a cyclic permutation of $\Pi_{i}, i=1,2,3$. The product $P Q$ is also in $S(\Pi)$ and hence $(P Q)^{3}=$ $K_{1} K_{2} K_{3}$ where each $K_{i}$ is a permutation of $\Pi_{i}$. Moreover, each orbit of $P Q$ is the union of an orbit of $K$ with an orbit of $K_{2}$ and one of $K_{3}$; hence $\|P Q\|=\left\|K_{1}\right\|$. Now,

$$
\begin{aligned}
K_{1} & =\left.(P Q P Q P Q)\right|_{\Pi_{1}}=\left.\left(P \tau_{2} P \tau_{3} P \tau_{1}\right)\right|_{\Pi_{1}} \\
& =\left.\left.\left.P^{3}\right|_{\Pi_{1}} \circ\left(P^{-2} \tau_{2} P^{2}\right)\right|_{\Pi_{1}} \circ\left(P^{-1} \tau_{3} P\right)\right|_{\Pi_{1}} \circ \tau_{1} .
\end{aligned}
$$

Note that as $\tau_{2}$ and $\tau_{3}$ vary over all the cyclic permutations of $\Pi_{2}$ and $\Pi_{3}$, respectively, $\left.\left(P^{-2} \tau_{2} P^{2}\right)\right|_{\Pi_{1}}$ and $\left.\left(P^{-1} \tau_{3} P\right)\right|_{\Pi_{1}}$ each vary over all the cyclic permutations of $\Pi_{1}$. Hence, by Proposition 9 , it is possible to choose the $\tau_{i}$ (thus determining $Q$ ) so that

$$
\left\|K_{1}\right\|>n / 3-1=\omega-1
$$

Hence,

$$
\begin{aligned}
\mu(P, \Pi) & \geqslant\|P Q\|=\left\|K_{1}\right\|>\omega-1 \\
& >n-\kappa(P, \Pi)-\frac{1}{2} \sigma(P, \Pi)-\frac{1}{2} \omega(P, \Pi)-3 .
\end{aligned}
$$

2. Applications to topological graph theory. Our graph theoretical terminology agrees with that of [BC], except in that graphs can have loops and multiple edges. The set of arcs (directed edges) which have the vertex $u$ as their initial point is labeled $D_{u}$ and referred to as the arcs at $u$. A rotation system $R$ of the graph $G$ is a set of cyclic permutations $\left\{R_{u} \mid u \in V(G)\right\}$ where each $R_{u}$ acts on $D_{u}$. The edges of $G$ define an involution $E(G): \kappa_{1} \ldots \kappa_{q}$ on $D(G)=\cup\left\{D_{u} \mid u \in V(G)\right\}$ where each $\kappa_{i}$ is the transposition mapping each arc onto its inverse. Identify each rotation $R$ of $G$ with the product

$$
R: R_{v_{1}} R_{v_{2}} \ldots R_{v_{p}} \quad \text { where } V(G)=\left\{v_{1}, \ldots, v_{p}\right\}
$$


We then have the following well-known theorem.

THEOREM 14 [Y]. The oriented 2-cell embeddings of the connected graph $G$ are in a one-to-one correspondence with the rotation systems of $G$. The correspondence is such that if $E=E(G)$ then the boundaries of the regions of an embedding consist of the orbits of $E \circ R$ where $R$ is the associated rotation system.

Henceforth the graph $G$ will be assumed to be fixed. This also fixes $E=E(G)$ as an involution on the set $D(G)$. Observe that the rotation systems on $G$ can be identified with $S(\Pi)$ where $\Pi$ is the partition $\left\{D_{u} \mid u \in V(G)\right\}$ of $D(G)$.

Now let $U$ be a subset of the $V(G)$, and denote by $\partial(U)$ the set of all arcs at $U$ whose terminal point is not in $U$. Any rotation system will induce two permutations, $\operatorname{Ext}=\operatorname{Ext}(R, U)$ and $\operatorname{Int}=\operatorname{Int}(R, U)$ on $\partial(U)$ as follows. For any $d \in$ $\partial(U)$

$$
d \mathrm{Ext}=d(E R)^{x(d)} E, \quad d \mathrm{Int}=d(R E)^{n(d)} E,
$$

where $x(d)$ and $n(d)$ are the least positive integers such that $d(E R)^{x(d)} E \in D_{u}$ for some $u \in U$ and $(d)(R E)^{n(d)} \in D_{v}$ for some $v \notin U$. We now show that Ext is indeed a permutation of $\partial(U)$, leaving the analogous proof for Int to the reader. By definition, $d$ Ext $\in D_{u}$ for some $u \in U$. Moreover, ( $d$ Ext) $E$ has the same initial point, say $v$, as $(d E x t) E R^{-1}=d(E R)^{x(d)-1} E$. The minimality of $x(d)$ implies that $v \notin U$ and hence $d$ Ext $\in \partial(U)$. To show that Ext is injective, assume that $d$ Ext $=e$ Ext for some $d, e \in \partial(U)$. By symmetry we may suppose that $x(d)>$ $x(e)$. Hence, $d(E R)^{x(d)-x(e)-1} E=e R^{-1}$. However, the initial point of $e R^{-1}$ is the same as that of $e$ and is therefore in $U$. Since $x(d)-x(e)-1<x(d)$, this contradicts the minimality of $x(d)$. Thus, Ext is in fact a permutation of $\partial(U)$.

These two permutations actually have a very natural visual interpretation. Suppose that in the embedding of $G$ determined by $R$, the arc $d \in \partial(U)$ is contained in the oriented boundary of the region $\rho$ and that $d^{-1}$ is in the oriented boundary $\rho^{\prime}$. Starting from $d$, follow along the boundary of $\rho$ until the first edge belonging to $\partial(U)$ is encountered. This edge directed from $U$ to $V-U$ is $d$ Ext. Starting from $d^{-1}$, follow along the boundary of $\rho^{\prime}$ until the first edge belonging to $\partial(U)$ in encountered. This edge, directed from $U$ to $V-U$ is $d$ Int. It is clear from this interpretation that

$$
\begin{aligned}
& \operatorname{Ext}(R, U)=E \circ \operatorname{Int}(R, V-U) \circ E \text { and } \\
& \operatorname{Int}(R, U)=E \circ \operatorname{Ext}(R, V-U) \circ E .
\end{aligned}
$$

This can also be proved directly from the definition. The above interpretation also motivates the choice of symbols Ext and Int. The permutation Ext is determined by following arcs along the portions of the regions in the "exterior" of $U$; Int is defined by using the portions in the "interior" of $U$.

EXAMPLE 15. Let $G$ be the graph of Figure 1 with $R$ defined by the counterclockwise sense on the page. The reader is reminded that in this case, regions lie to the right of their oriented boundary. Set $U=\{1,2,3,4,5,6\}$; then $\partial(U)=$ $\{47,48,58,68\} ; \operatorname{Ext}(R, U)=(47685848), \operatorname{Int}(R, U)=(47485868)$. 


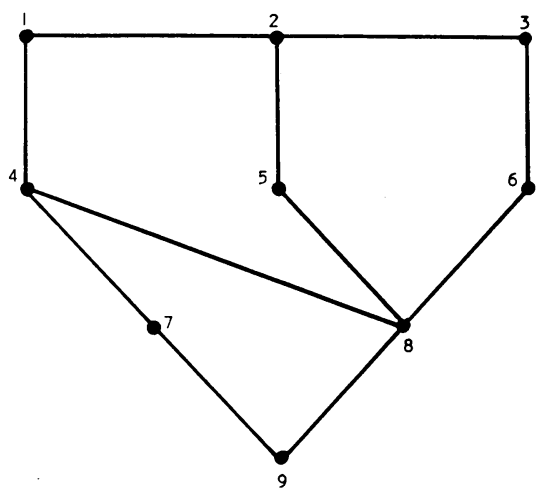

Figure 1

EXAmple 16. Let $G$ be the graph of Figure 2 with $R$ again defined by the counterclockwise sense. Set $U=\{1\}$. Then $\partial(U)=\{12,13,15,16\}, \operatorname{Ext}(R, U)=$ (13 12)(15 16), $\operatorname{Int}(R, U)=(121516$ 13). The following theorem has proven to be very useful in that it makes it possible to ignore the action of a rotation system on all but a selected subset of the arcs of a graph.

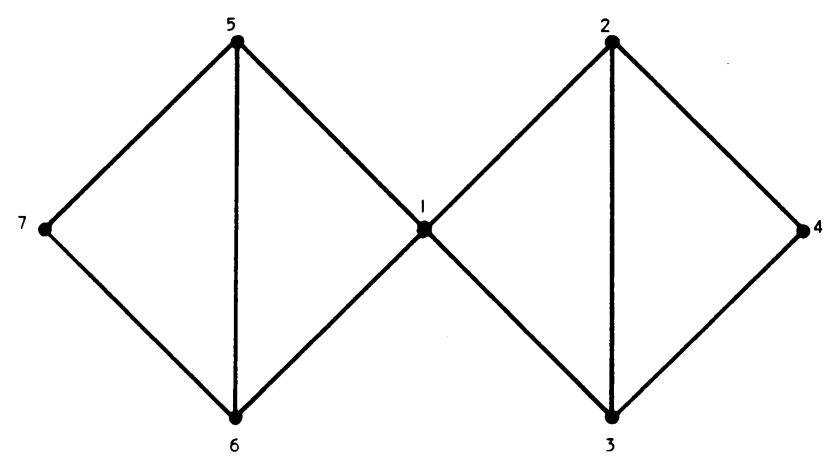

Figure 2

THEOREM 17. Let $G$ be a graph, $R$ a rotation system of $G$ and $U \subseteq V(G)$. Then the number of regions determined by $R$ and containing edges of $\partial(U)$ is $\|\operatorname{Ext}(R, U) \circ \operatorname{Int}(R, U)\|$.

Proof. If $d \in \partial(U)$, then, if $e=d \operatorname{Ext}(R, U)$ we have

$$
\begin{aligned}
d \operatorname{Ext}(R, U) \circ \operatorname{Int}(R, U) & =e \operatorname{Int}(R, U)=e(R E)^{n(e)} E \\
& =d(E R)^{x(d)} E(R E)^{n(e)} E=d(E R)^{x(d)+n(e)} .
\end{aligned}
$$

It follows from the definition of $n(e)$ that $d(E R)^{x(d)+n(e)} \in \partial(U)$. We now show that $x(d)+n(e)$ is in fact the least such positive integer. For suppose $d(E R)^{m} \in$ $\partial(U)$ for some positive integer $m$. Since $d \in \partial(U)$, it follows that $d E R \in D_{u}$ for some $u \notin U$. But $x(d)$ is the least positive integer such that $d(E R)^{x(d)} E R \in D_{u}$ for some $u \in U$, because the application of the last $R$ does not change initial vertices. Hence, $x(d)<m$. Now, since $d(E R)^{m} \in \partial(U)$, we have

$$
d(E R)^{m} E \in D_{u} \quad \text { for some } u \notin U,
$$


or

$$
d(E R)^{x(d)} E(E R)^{m-x(d)} \in D_{u} \quad \text { for some } u \notin U .
$$

However, by definition, $e=d$ Ext $=d(E R)^{x(d)} E$ and hence

$$
e(R E)^{m-x(d)} \in D_{u} \quad \text { for some } u \notin U .
$$

Consequently, $m-x(d) \geqslant n(e)$ or $m \geqslant x(d)+n(e)$, thus proving the minimality of $x(d)+n(e)$.

It follows from the above proven minimality that the orbits of $\operatorname{Ext}(R, U) \circ \operatorname{Int}(R, U)$ are obtained from those of the global permutation $E R$ by deleting all the arcs not in $\partial(U)$. The statement of the theorem now follows immediately.

Given the set up of the above theorem it is convenient to define $r(R, U)$ as the number of regions of the embedding determined by $R$, that contain $\operatorname{arcs}$ of $\partial(U)$. It is clear that $r(R, U)=r(R, V-U)$. We define $r(R)$ as the total number of regions of the embedding determined by $R$.

In most of the subsequent theorems, the construction of a certain optimal rotation system $R$ is called for. In all these cases, a certain subset $U \subseteq V(G)$ is given and $R$ is first specified in its complement $V(G)-U$. Then, without specifying $R$ or $U$, conclusions are drawn about $\operatorname{Ext}(R, U)$. We draw the reader's attention to the fact that $\operatorname{Ext}(R, U)$ is in fact independent of the values of $R$ at vertices of $U$.

Let $G^{i}, i=1,2$, be two graphs with subgraphs $H^{i}$ which are isomorphic to some graph $H$. Let $G$ be the graph obtained from $G^{1}$ and $G^{2}$ by identifying the corresponding vertices and edges in the $H^{i}$. We write $G=G^{1} \vee_{H} G^{2}$ and refer to this process as an amalgamation.

EXAMPLE 18.

$$
G^{1}=\underbrace{9}_{G=G^{1} \vee_{H} G^{2}=}, G^{2}=\underbrace{\infty}_{0},
$$

EXAMPLE 19.

$$
\begin{aligned}
& G^{1}=\alpha \int_{0}^{0}, \quad G^{2}=\underbrace{0}_{0}, \\
& G=G^{1} \vee_{H} G^{2}=
\end{aligned}
$$


These amalgamations were first studied in depth by S. Alpert [A1], [A2]. The following theorem was recently, and independently, proved by Decker, Glover, and Huneke in [DGH]. Their proof is quite different from the one given here, although similarities do exist between some of the concepts involved.

THEOREM 20. Let $G, G^{1}$ and $G^{2}$ be connected graphs such that $G=G^{1} \vee{ }_{U} G^{2}$ where $U=\{1,2\}$ and $12 \notin E(G)$. Then

$$
\gamma\left(G^{1}\right)+\gamma\left(G^{2}\right)+1 \geqslant \gamma(G)>\gamma\left(G^{1}\right)+\gamma\left(G^{2}\right)-1 .
$$

Proof. To prove the upper bound, one need merely start with genus embeddings of $G^{1}$ and $G^{2}$ and connect them with two tubes, one joining the two images of 1 and the other joining the two images of 2 .

To prove the lower bound, suppose $R$ is a rotation system which determines a genus embedding of $G$. Define $R_{w}^{i}=R_{w}$ whenever $w \in V\left(G^{i}\right)-U, i=1,2$, and set $\operatorname{Ext}=\operatorname{Ext}(R, U)$ as well as $\operatorname{Ext}^{i}=\operatorname{Ext}\left(R^{i}, U\right)$. Note that since $12 \notin U$, it follows that $\operatorname{Int}(R, U)=R_{1} \circ R_{2}$. Hence, by Theorem 17,

$$
r(R, U)=\| \text { Ext } \circ \operatorname{Int}(R, U)\|=\| \text { Ext } \circ R_{1} \circ R_{2} \| .
$$

Moreover, since genus embeddings maximize the number of regions,

$$
r(R, U)=\mu\left(\operatorname{Ext},\left\{D_{j}(G)\right\}_{j=1}^{2}\right)
$$

Similarly, we may define the $R_{j}^{i}, j=1,2$, so that

$$
r\left(R^{i}, U\right)=\mu\left(\operatorname{Ext}^{i},\left\{D_{j}\left(G^{i}\right)\right\}_{j=1}^{2}\right), \quad i=1,2 .
$$

Since the set $U$ separates $G^{1}-U$ from $G^{2}-U$, it follows that Ext is the disjoint product $\operatorname{Ext}^{1} \circ \operatorname{Ext}^{2}$. If the $\operatorname{PP}(\cdot, 2)$ pair $\left(\operatorname{Ext},\left\{D_{j}(G)\right\}_{j=1}^{2}\right)$ is decomposable, so are the pairs $\left(\operatorname{Ext}^{i},\left\{D_{j}\left(G^{i}\right)\right\}_{j=1}^{2}\right)$. It then follows from Corollary 8 that

$$
\begin{aligned}
r(R, U) & =\left|D_{1}(G)\right|+\left|D_{2}(G)\right|-\|\mathrm{Ext}\|+2 \\
& =\sum_{i=1}^{2}\left(\left|D_{1}\left(G^{i}\right)\right|+\left|D_{2}\left(G^{i}\right)\right|-\left\|\operatorname{Ext}^{i}\right\|+2\right)-2 \\
& =r\left(R^{1}, U\right)+r\left(R^{2}, U\right)-2 .
\end{aligned}
$$

If, on the other hand, (Ext, $\left.\left\{D_{j}(G)\right\}_{j=1}^{2}\right)$ is indecomposable, then, with a slight abuse of notation, by Theorem 10 ,

$$
\begin{aligned}
r(R, U) & <\left|D_{1}(G)\right|+\left|D_{2}(G)\right|-\kappa-\frac{1}{2} \sigma \\
& =\sum_{i=1}^{2}\left(\left|D_{1}\left(G^{i}\right)\right|+\left|D_{2}\left(G^{i}\right)\right|-\kappa^{i}-\frac{1}{2} \sigma^{i}-1\right)+2 .
\end{aligned}
$$

So, if both (Ext $\left.{ }^{i},\left\{D_{j}\left(G^{i}\right)\right\}_{j=1}^{2}\right)$ are also indecomposable, we conclude that

$$
r(R, U)<r\left(R^{1}, U\right)+r\left(R^{2}, U\right)+2 .
$$

If one or both of these pairs is decomposable, observe that

$$
\left|D_{1}\left(G^{i}\right)\right|+\left|D_{2}\left(G^{i}\right)\right|-\kappa^{i}-\frac{1}{2} \sigma^{i}-1<\left|D_{1}\left(G^{i}\right)\right|+\left|D_{2}\left(G^{i}\right)\right|-\left\|\operatorname{Ext}^{i}\right\|+2
$$

and hence inequality (6) holds again. In fact we have shown that this inequality holds in all cases. The theorem now follows from the Euler-Poincare formula in the 
following manner. First we note that the regions of $R$ which do not contain any points of $U$ are passed intact to either $R^{1}$ or $R^{2}$ but not both. Hence it follows from (6) that

$$
r(R)-2 \leqslant r\left(R^{1}\right)+r\left(R^{2}\right) .
$$

Now,

$$
\begin{aligned}
\gamma\left(G^{1}\right)+\gamma\left(G^{2}\right)-1 \leq & \frac{1}{2}\left(-V\left(G^{1}\right)+E\left(G^{1}\right)-r\left(R^{1}\right)+2\right) \\
& +\frac{1}{2}\left(-V\left(G^{2}\right)+E\left(G^{2}\right)-r\left(R^{2}\right)+2\right)-1 \\
< & \frac{1}{2}(-|V(G)|-2+|E(G)|-r(R)+2+4-2) \\
= & \frac{1}{2}(-2+2 \gamma(G)+2)=\gamma(G) . \quad \square
\end{aligned}
$$

Corollary 21. If $G=G_{1} \bigvee_{K_{2}} G_{2}$, then

$$
\gamma\left(G_{1}\right)+\gamma\left(G_{2}\right)>\gamma(G)>\gamma\left(G_{1}\right)+\gamma\left(G_{2}\right)-1 .
$$

Proof. The upper bound has a succinct proof in [A1]. The lower bound is proved by subdividing the common edge of $G_{1}$ and $G_{2}$ and applying Theorem 20.

TheOREM 22. Let $G, G^{1}$ and $G^{2}$ be connected graphs such that $G=G^{1} \vee_{U} G^{2}$ where $U=\{1,2,3\}$ and $12,23,13 \notin E(G)$.

Then

$$
\gamma\left(G^{1}\right)+\gamma\left(G^{2}\right)+2>\gamma(G)>\gamma\left(G^{1}\right)+\gamma\left(G^{2}\right)-2 .
$$

Proof. The upper bound is again proved by tube addition.

For the lower bound let $R$ be a rotation system defining a genus embedding of $G$. Set $R_{w}^{i}=R_{w}$ whenever $w \in V\left(G^{i}\right)-U, i=1,2$ and $\operatorname{Ext}=\operatorname{Ext}(R, U), \operatorname{Ext}^{i}=$ $\operatorname{Ext}\left(R^{i}, U\right)$. As in the previous theorem, $R_{j}^{i}, j=1,2,3$, may be defined so that

$$
\begin{aligned}
& r(R, U)=\mu\left(\operatorname{Ext},\left\{D_{j}(G)\right\}_{j-1}^{3}\right), \\
& r\left(R^{i}, U\right)=\mu\left(\operatorname{Ext}^{i},\left\{D_{j}\left(G^{i}\right)\right\}_{j=1}^{3}\right), \quad i=1,2 .
\end{aligned}
$$

Here too, Ext is the disjoint product $\operatorname{Ext}^{1} \circ \operatorname{Ext}^{2}$. If the $\operatorname{PP}(\cdot, 3)$ pair (Ext, $\left.\left\{D_{j}(G)\right\}_{j=1}^{3}\right)$ is indecomposable, then

$$
\begin{aligned}
r(R, U) & <\sum_{j=1}^{3}\left|D_{j}(G)\right|-\kappa-\frac{1}{2} \sigma-\frac{1}{2} \omega \\
& =\sum_{i=1}^{2} \sum_{j=1}^{3}\left(\left(\left|D_{j}(G)\right|\right)-\kappa^{i}-\frac{1}{2} \sigma^{i}-\frac{1}{2} \omega^{i}-3\right)+6 .
\end{aligned}
$$

Observe that for any $P P(n, 3)$ pair,

$$
n-\kappa-\frac{1}{2} \sigma-\frac{1}{2} \omega-3<n-\kappa-\frac{1}{2} \sigma+1<n-\kappa+3 .
$$

Hence, invoking Theorem 13 and Corollary 11, and applying them to (7), it follows that, regardless of the possible decomposability of $\left(\operatorname{Ext}^{i},\left\{D_{j}\left(G^{i}\right)\right\}_{j=1}^{3}\right)$,

$$
r(R, U)<r\left(R^{1}, U\right)+r\left(R^{2}, U\right)+6 .
$$

Should (Ext, $\left\{D_{j}(G)\right\}_{j=1}^{3}$ ) be decomposable, we note that its decomposition induces 
an analogous decomposition on both pairs $\left(\operatorname{Ext}^{i},\left\{D_{j}\left(G^{i}\right)\right\}_{j=1}^{3}\right), i=1,2$, and inequality (8) can be verified in the same manner as was done in the proof of Theorem 22. The details are omitted.

An application of the Euler-Poincaré formula to (8) yields the inequality

$$
2 \gamma(G) \geqslant 2 \gamma\left(G^{1}\right)+2 \gamma\left(G^{2}\right)-5 \text {. }
$$

Since the genus parameter is integer valued, the statement of the theorem now follows.

The following theorem first appeared in [HK]. The proof given there employs the Mayer-Vietoris sequence.

TheOREM 23. Let $G, G^{1}$ and $G^{2}$ be connected graphs such that $G=G^{1} \vee_{U} G^{2}$ where $U$ is independent. If $\gamma(G+u v)=\gamma(G)+1$ for any distinct points $u$, $v$ of $U$, then

$$
\gamma(G)=\gamma\left(G^{1}\right)+\gamma\left(G^{2}\right)+|U|-1 .
$$

Proof. Suppose $U=1,2, \ldots, k$. If $R^{i}$ is a rotation system of $G^{i}, i=1,2$, then since $U$ is independent,

$$
\operatorname{Int}\left(R^{i}, U\right)=\prod_{j=1}^{k} R_{j}^{i}, \quad i=1,2 .
$$

Now suppose that $R$ is a rotation system which determines a genus embedding of $G$. Define $R_{v}^{i}=R_{v}$ whenever $v \in V\left(G^{i}\right)-U, i=1,2$. The values of $R^{\mathrm{i}}$ at vertices of $U$ will be specified later. Observe that the condition $\gamma(G+u v)=\gamma(G)+1$ for distinct $u, v \in U$, implies that every region of $R$ contains arcs of at most one vertex of $U$. Consequently, each orbit of $\operatorname{Ext}=\operatorname{Ext}(R, U)$ is completely contained in one of the $D_{j}$, as well as in either $G^{1}$ or $G^{2}$. Let $\operatorname{Ext}_{j}^{i}, j=1,2, \ldots, k, i=1,2$, be the product of those cyclic factors of Ext contained in $D_{j}\left(G^{i}\right)$. Set $\operatorname{Ext}_{j}=\operatorname{Ext}_{j}^{1} \circ \mathrm{Ext}_{j}^{2}$ and $\mathrm{Ext}^{i}=\Pi_{j=1}^{k} \mathrm{Ext}_{j}^{i}$. The above observations imply that the pair (Ext, $\left.\left\{D_{j}(G)\right\}_{j=1}^{k}\right)$ is decomposable into $\left\{\left(\operatorname{Ext}_{j},\left\{D_{j}(G)\right\}\right)\right\}_{j=1}^{k}$ while $\left(\operatorname{Ext}^{i},\left\{D_{j}\left(G^{i}\right)\right\}_{j=1}^{k}\right)$ decomposes into $\left\{\left(\operatorname{Ext}_{j}^{i},\left\{D_{j}\left(G^{i}\right)\right\}\right)\right\}_{j=1}^{k}$. Hence, by Corollary 8 ,

$$
\begin{aligned}
\mu\left(\operatorname{Ext},\left\{D_{j}(G)\right\}_{j=1}^{k}\right) & =\sum_{j=1}^{k}\left|D_{j}(G)\right|-\|\operatorname{Ext}\|+k, \\
\mu\left(\operatorname{Ext}^{i},\left\{D_{j}\left(G^{i}\right)\right\}_{j=1}^{k}\right) & =\sum_{j=1}^{k}\left|D_{j}\left(G^{i}\right)\right|-\left\|\operatorname{Ext}^{i}\right\|+k, \quad i=1,2 .
\end{aligned}
$$

However, $\sum_{j=1}^{k}\left|D_{j}(G)\right|=\sum_{i=1}^{2} \sum_{j=1}^{k}\left|D_{j}\left(G^{i}\right)\right|$ and $\|\operatorname{Ext}\|=\left\|\operatorname{Ext}^{1}\right\|+\left\|\operatorname{Ext}^{2}\right\|$ since $\mathrm{Ext}^{1}$ and $\mathrm{Ext}^{2}$ are disjoint permutations. Hence,

$$
\mu\left(\operatorname{Ext},\left\{D_{j}(G)\right\}_{j=1}^{k}\right)=\sum_{i=1}^{2} \mu\left(\operatorname{Ext}^{i},\left\{D_{j}\left(G^{i}\right)\right\}_{j=1}^{k}\right)-k .
$$

Specify $R^{1}$ and $R^{2}$ on $U$ so that

$$
\begin{aligned}
r\left(R^{i}, U\right) & =\left\|\operatorname{Ext}\left(R^{i}, U\right) \circ \operatorname{Int}\left(R^{i}, U\right)\right\|=\left\|\operatorname{Ext}^{i} \circ \prod_{j=1}^{k} R_{j}^{i}\right\| \\
& =\mu\left(\operatorname{Ext}^{i},\left\{D_{j}\left(G^{i}\right)\right\}_{j=1}^{k}\right) .
\end{aligned}
$$


Since genus embeddings maximize the number of regions, we already know that $r(R, U)=\mu\left(\operatorname{Ext},\left\{D_{j}(G)\right\}_{j=1}^{k}\right)$. It now follows from (9) above that

$$
r(R, U)=r\left(R^{1}, U\right)+r\left(R^{2}, U\right)-k .
$$

A straightforward application of the Euler-Poincaré formula yields the inequality

$$
\gamma(G) \geqslant \gamma\left(G^{1}\right)+\gamma\left(G^{2}\right)+k-1 .
$$

The reverse inequality can be proved by connecting genus embeddings of $G^{1}$ and $G^{2}$ with $k$ appropriately placed tubes.

The following corollary is the celebrated Battle, Harary, Kodoma, and Youngs Theorem [BHKY]. It is proved by setting $k=1$ in the previous theorem and then applying induction to $n$.

COROLlaRY 24. If $B_{1}, \ldots, B_{n}$ are the blocks of the connected graph $G$, then $\gamma(G)=\sum_{i=1}^{n} \gamma\left(B_{i}\right)$.

We now turn to the maximum genus $\gamma^{M}(G)$. The following theorem appears in [LR], [Y]. The proof given here is similar to that of [LR].

THEOREM 25. If $u$ is a vertex of $G$ and $G=G^{1} \bigvee_{\{u\}} G^{2}$ is a connected graph, then $\gamma^{M}(G)=\gamma^{M}\left(G^{1}\right)+\gamma^{M}\left(G^{2}\right)+\delta$ where $\delta \in\{0,1\}$. The value $\delta=1$ holds if and only if both $G_{1}$ and $G_{2}$ have maximum genus embeddings in which $u$ appears on the boundary of two regions.

Proof. Let $R$ be a rotation system defining a maximum genus embedding of $G$. Define $R_{v}^{i}=R_{v}$ whenever $v \in V\left(G^{i}\right)-\{u\}, i=1$, 2. It follows from Proposition 9c that $R_{u}^{i}=\operatorname{Int}\left(R^{i},\{u\}\right)$ can be specified so that

$$
r\left(R^{i},\{u\}\right)=\left\|\operatorname{Ext}\left(R^{i},\{u\}\right) \circ \operatorname{Int}\left(R^{i},\{u\}\right)\right\|<2, \quad i=1,2 .
$$

Since $r(R,\{u\}) \geqslant 1$ it follows that $r\left(R^{1},\{u\}\right)+r\left(R^{2},\{u\}\right)<r(R,\{u\})+3$ and hence

$$
r\left(R^{1}\right)+r\left(R^{2}\right)<r(R)+3 .
$$

The Euler-Poincare formula now yields the inequality,

$$
\gamma^{M}\left(G^{1}\right)+\gamma^{M}\left(G^{2}\right)+1>\gamma^{M}(G) .
$$

Observe that if equality holds in (11), then it must also hold in (10) and so $R^{1}$ and $R^{2}$ determine maximum genus embeddings in which

$$
r\left(R^{1},\{u\}\right)=r\left(R^{2},\{u\}\right)=2 .
$$

Suppose that $R^{i}$ is a rotation system which determines a maximum genus embedding for $G^{i}, i=1,2$. Define $R_{v}=R_{v}^{i}$ whenever $u \in V\left(G^{i}\right)-\{u\}$, and choose $R_{u}$ so that

$$
r(R,\{u\})=\|\operatorname{Ext}(R,\{u\}) \circ \operatorname{Int}(R,\{u\})\|<2 .
$$

Since $r\left(R^{i},\{u\}\right)>1$ for $i=1,2$, it follows that $r\left(R^{1},\{u\}\right)+r\left(R^{2},\{u\}\right)>$ $r(R,\{u\})$ and hence $r\left(R^{1}\right)+r\left(R^{2}\right)>r(R)$. From this the inequality $\gamma^{M}\left(G^{1}\right)+$ $\gamma^{M}\left(G^{2}\right)>\gamma^{M}(G)$ follows immediately.

If $R^{i}$ has the additional property that $r\left(R^{i},\{u\}\right)=2$ for $i=1,2$, then $r\left(R^{1}\right)+$ $r\left(R^{2}\right)>r(R)+2$ and so $\gamma^{M}(G)>\gamma^{M}\left(G^{1}\right)+\gamma^{M}\left(G^{2}\right)+1$. 
COROLlaRY 26 [NRSW]. If the edge e of the connected graph $G$ is a bridge and if $G_{1}$ and $G_{2}$ are the connected components of $G-e$, then

$$
\gamma^{M}(G)=\gamma^{M}\left(G_{1}\right)+\gamma^{M}\left(G_{2}\right)
$$

Proof. First apply Theorem 25 to $G_{1}$ and $e$, so that $\gamma^{M}\left(G_{1} \cup e\right)=\gamma^{M}\left(G_{1}\right)$. Then apply the same theorem to $G_{2}$ and $G_{1} \cup e$, observing that in any embedding of $G_{1} \cup e$, the "free" end of $e$ is on the boundary of exactly one region.

The following theorem was first proved in [NRSW].

THEOREM 27. The connected graph $G$ satisfies the condition $\gamma(G)=\gamma^{M}(G)$, if and only if every vertex of $G$ is contained in at most one cycle.

Proof. Suppose $\gamma(G)=\gamma^{M}(G)$, and let $v$ be any vertex of $G$. If $R^{1}$ and $R^{2}$ are any two rotation systems on $G$ which differ only at $v$, then clearly $\operatorname{Ext}\left(R^{1}\right.$, $\{v\})=\operatorname{Ext}\left(R^{2},\{v\}\right)=$ Ext. Now choose $R_{v}^{1}$ so as to maximize $r\left(R^{1},\{v\}\right)=$ $\|$ Ext $\circ R_{v}^{1} \|$ and choose $R_{v}^{2}$ so as to minimize $r\left(R^{2},\{v\}\right)=\|$ Ext $\circ R_{v}^{2} \|$. Since $\gamma(G)=\gamma^{M}(G)$, it follows that $r\left(R^{1},\{v\}\right)=r\left(R^{2},\{v\}\right)$ and hence, by Theorem 7 and Proposition 9c,

$$
\left|D_{v}\right|-\|\operatorname{Ext}\|+1=\mu\left(\operatorname{Ext},\left\{D_{v}\right\}\right)=r\left(R^{1},\{v\}\right)=r\left(R^{2},\{v\}\right)<2
$$

or

$$
\|\mathrm{Ext}\|>\operatorname{deg}_{u}-1 \text {. }
$$

Now if $G$ contains a point $u$ on two cycles, then it clearly contains a subgraph homeomorphic from the graphs of Figure 3. If $G \supseteq H$, let $R$ be any rotation system of $G$ such that

$$
R_{u}=(\operatorname{def} \ldots), \quad R_{v}=\left(f^{-1} e^{-1} d^{-1} \ldots\right) .
$$

Then $\operatorname{Ext}=\operatorname{Ext}(R,\{u\})$ has as one of its orbits $($ def $\ldots)$ and hence $\|\operatorname{Ext}\|<\operatorname{deg}_{0}$ -2 , contradicting (12) above. If $G$ contains a subgraph homeomorphic from $H$ or $H^{\prime}$, then $R$ is defined in a similar manner and leads to the same contradiction.

Conversely, if every vertex of $G$ is on at most one cycle, then after removing its bridges, all that remains of $G$ is a set of disjoint cycles, each of which has $\gamma=\gamma^{M}=0$.

By Corollaries 24 and 26, $\gamma(G)=\gamma^{M}(G)$.

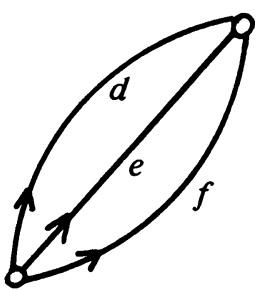

H

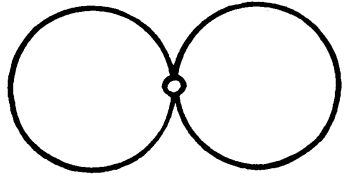

$H^{\prime}$

Figure 3 
Conclusion. Theorems $20-24$ indicate that the genus parameter is approximately additive over the amalgamation of graphs. This is formalized in the following conjecture where $Z^{+}$denotes the set of positive integers.

Conjecture 28. There exists a function $f: Z^{+} \rightarrow Z^{+}$such that for any graphs $G$ and $H$,

$$
\gamma\left(G \vee_{K} H\right)>\gamma(G)+\gamma(H)-f(|V(K)|) .
$$

This function, if it exists, must have quadratic order, as is indicated by the case $G=H=K=K_{n}$, where $K_{n}$ denotes the complete graph on $n$ vertices.

ACKNowledgement. The author thanks Seth Alpert, Evelyn Boorman, and Fred Galvin for helping him get unstuck at various stages of the research leading to this paper.

\section{BIBLIOGRAPHY}

[A1] S. R. Alpert, The genera of amalgamations of graphs, Trans. Amer. Math. Soc. 78 (1973), 1-39. [A2] _ The genera of edge amalgamations of complete bigraphs (in preparation).

[BC] M. Behzad and G. Chartrand, Introduction to the theory of graphs, Allyn and Bacon, Boston, Mass., 1971.

[BHKY] J. Battle, F. Harary, Y. Kodama and J. W. T. Youngs, Additivity of the genus of a graph, Bull. Amer. Math. Soc. 68 (1962), 565-568.

[C] A. Cayley, On the colouring of maps, Proc. London Math. Soc. 9 (1878), 148.

[DGH] R. W. Decker, H. H. Glover and J. P. Huneke, The genus of 2-connected graphs (in preparation).

[HK] F. Harary and Y. Kodama, On the genus of an n-connected graph, Fund. Math. 54 (1964), 7-13.

[H1] P. J. Heawood, Map colour theorem, Quart. J. Math. 24 (1890), 332-338.

[H2] D. Husemoller, Ramified coverings of Riemann surfaces, Duke Math. J. 29 (1962), 167-174.

[LR] C. Little and R. Ringeisen, An additivity theorem for maximum genus of a graph, Discrete Math. 21 (1978), 69-74.

[NRSW] E. A. Nordhaus, R. D. Ringeisen, B. M. Stewart and A. T. White, A Kuratowski type theorem for the maximum genus of a graph, J. Combinatorial Theory Ser. B 12 (1972), 260-267.

[R] G. Ringel, Map color theorem, Springer-Verlag, Berlin and New York, 1974.

[S] S. Stahl, A counting theorem for topological graph theory, Theory and Application of Graphs (Proc. Conf. Western Michigan Univ., Kalamazoo, Mich., 1976), Y. Alavi and D. R. Lick (eds.), Lecture Notes in Math., vol. 642, Springer-Verlag, Berlin and New York, 1976, pp. 534-544.

[W] T. R. S. Walsh, Hypermaps versus bipartite maps, J. Combinatorial Theory Ser. B 18 (1975), $155-163$.

[X] N. H. Xuong, Sur les immersions d'un graphe dans les surfaces orientably, C. R. Acad. Sci. Paris 283 (1976).

[Y] J. W. T. Youngs, Minimal imbeddings and the genus of a graph, J. Math. Mech. 12 (1963), 303-316.

Departiment of MATHEMatics, University of Kansas, LAWrence, Kansas 66045 